\title{
Breaking through the false coincidence barrier in electron-ion coincidence experiments
}

\author{
David L. Osborn, ${ }^{1}$ Carl C. Hayden, ${ }^{1}$ Patrick Hemberger, ${ }^{2}$ Andras Bodi, ${ }^{2}$ \\ Krisztina Voronova, ${ }^{3}$ and Bálint Sztáray ${ }^{3, a)}$ \\ ${ }^{1}$ Combustion Research Facility, Sandia National Laboratories, Livermore, California 94551, USA \\ ${ }^{2}$ Laboratory for Femtochemistry and Synchrotron Radiation, Paul Scherrer Institute, Villigen 5232, Switzerland \\ ${ }^{3}$ Department of Chemistry, University of the Pacific, Stockton, California 95211, USA
}

(Received 3 September 2016; accepted 6 October 2016; published online 31 October 2016)

\begin{abstract}
Photoelectron Photoion Coincidence (PEPICO) spectroscopy holds the promise of a universal, isomer-selective, and sensitive analytical technique for time-resolved quantitative analysis of bimolecular chemical reactions. Unfortunately, its low dynamic range of $\sim 10^{3}$ has largely precluded its use for this purpose, where a dynamic range of at least $10^{5}$ is generally required. This limitation is due to the false coincidence background common to all coincidence experiments, especially at high count rates. Electron/ion pairs emanating from separate ionization events but arriving within the ion time of flight (TOF) range of interest constitute the false coincidence background. Although this background has uniform intensity at every $\mathrm{m} / \mathrm{z}$ value, the Poisson scatter in the false coincidence background obscures small signals. In this paper, temporal ion deflection coupled with a position-sensitive ion detector enables suppression of the false coincidence background, increasing the dynamic range in the PEPICO TOF mass spectrum by $2-3$ orders of magnitude. The ions experience a time-dependent electric deflection field at a well-defined fraction of their time of flight. This deflection defines an $\mathrm{m} / \mathrm{z}-$ and ionization-time dependent ion impact position for true coincidences, whereas false coincidences appear randomly outside this region and can be efficiently suppressed. When cold argon clusters are ionized, false coincidence suppression allows us to observe species up to $\mathrm{Ar}_{9}{ }^{+}$, whereas $\mathrm{Ar}_{4}{ }^{+}$ is the largest observable cluster under traditional operation. This advance provides mass-selected photoelectron spectra for fast, high sensitivity quantitative analysis of reacting systems. Published by AIP Publishing. [http://dx.doi.org/10.1063/1.4965428]
\end{abstract}

\section{INTRODUCTION}

The ideal analytical experiment is universal (applicable to mixtures of all chemicals), selective (able to identify similar species unambiguously), sensitive (detecting intermediates with short lifetimes and/or low concentrations), and multiplexed (accomplishing these goals simultaneously). Gas chromatography coupled with mass spectrometry almost fits the bill for gas phase chemistry applications, but its time scale is much too slow to detect free radicals and short-lived reaction intermediates. By contrast, time-resolved multiplexed photoionization mass spectrometry (MPIMS) usually achieves all these goals and has been employed extensively over the past decade in the study of chemical reactions. ${ }^{1-3}$ Despite the modest spectral detail in photoionization (PI) spectroscopy, i.e., cation yield vs. photon energy, MPIMS is a powerful tool for rapid resolution of a mixture of neutral species first by ion mass-to-charge $(\mathrm{m} / \mathrm{z})$ ratio and second by photoionization spectrum within each $m / z$ channel.

When a cold sample is ionized by monochromatic vacuum ultraviolet (VUV) radiation, the parent ion yield in a PI spectrum begins at the ionization energy and increases with photon energy, often showing step-like features

\footnotetext{
a) Author to whom correspondence should be addressed. Electronic mail: bsztaray@pacific.edu
}

each time a new electronic or vibrational state of the photoion becomes energetically accessible. The onset and shape of the PI spectrum is a spectral fingerprint used to distinguish different neutral isomers observed at the same parent $\mathrm{m} / \mathrm{z}$ ratio. A more detailed spectral fingerprint can be measured with photoelectron spectroscopy, in which the electron ejected upon photoionization is detected instead. For each neutral species, the photoelectron intensity vs. electron binding energy yields the photoelectron (PE) spectrum. In contrast to the step-like thresholds that may be present when the photon energy is scanned to yield a PI spectrum, each quantum state of the photoion creates a peak in the photoelectron spectrum. Because the PE spectrum is primarily determined by the vibrational wave function overlap between the neutral and the cationic states, quantified by Franck-Condon factors, even similar isomers often yield distinct vibrational progressions. Even if the photoelectron spectrum consists of diffuse bands due to spectral congestion, the position and shape of the ground and excited electronic state bands are characteristic of the molecular structure. Furthermore, the vibrational envelopes can be calculated quite reliably, enabling a robust assignment of the signal, even for species with no experimental photoelectron spectrum. ${ }^{4,5}$ Unfortunately, the power of photoelectron spectroscopy suffers when a mixture of compounds is ionized, because the resulting photoelectron spectrum is the sum of the 
photoelectron spectra of all neutrals, regardless of their mass, making quantitative analysis difficult or impossible. By contrast, a PI spectrum acquired with a mass spectrometer is inherently mass resolved. Photoelectron photoion coincidence (PEPICO) spectroscopy combines photoelectron spectroscopy and mass spectrometry and holds the promise to merge the analytic benefits of MPIMS with the increased spectral information of photoelectron spectroscopy by recording simultaneous but separable photoelectron spectra for each $m / z$ ratio. $^{6}$

Mass analysis of cations, combined with kinetic energy analysis of electrons, can produce mass-resolved photoelectron spectra if the electrons and cations can be sorted into correlated pairs, each pair arising from the ionization of an individual neutral molecule. Such a correlated pair is called a true coincidence and requires detection of both charged particles. By contrast, an electron/cation pair in which the two particles did not arise from the same neutral molecule is termed a false coincidence. False coincidences contribute to the background signal of a PEPICO experiment and limit dynamic range, obscuring weak signals of trace species, free radicals, and reaction intermediates. Therefore, although PEPICO has proven to be extremely versatile in studying energetics, ${ }^{7}$ unimolecular dissociation mechanisms, ${ }^{8}$ and even quantum effects, ${ }^{9}$ its analytical applications have so far been limited by this false coincidence barrier. A number of pyrolysis $^{10,11}$ and flame experiments ${ }^{12}$ have succeeded using PEPICO detection, but even in these applications, greater dynamic range would be a clear benefit.

In this article, we use PEPICO to mass analyze cations via time-of-flight (TOF) mass spectrometry, augmented with new spatial dimensions of detection that enables the rejection of nearly all false coincidences. This new approach allows us to break through the false coincidence barrier, improving dynamic range by two to three orders of magnitude and enabling minor constituents of the sample to be detected, identified, and quantified via their photoelectron spectra. This enhancement makes PEPICO a viable method for universal quantitative analysis of gas phase reaction systems, including characterization of short-lived reactive intermediates in a variety of fields, such as combustion and atmospheric kinetics, flame chemistry, and heterogeneous catalysis.

\section{BACKGROUND}

In continuous PEPICO experiments, a static electric extraction field is used to accelerate the continuous flux of photoelectrons and photoions in opposite directions, as shown in Fig. 1(a). The $m / z$ of the photoion is derived from its time of flight. In contrast to pulsed laser ionization, the time of ionization cannot be known a priori with a continuous photon source. However, electrons are detected practically instantaneously, due to their low mass, and this signal can be used as the start trigger for the photoion TOF analysis on the microsecond time scale. Velocity map imaging ${ }^{13}$ of photoelectrons has been used in PEPICO experiments for a little more than a decade, ${ }^{14,15}$ dramatically improving electron kinetic energy resolution and collection efficiency. This advance has breathed new life into the technique, and several new PEPICO apparatuses have been commissioned at high flux synchrotron VUV photon sources. ${ }^{16-20}$ Using the full available flux of such beamlines results in ionization rates of $10^{5}-10^{6} \mathrm{~s}^{-1}$, corresponding to $1-10 \mu \mathrm{s}$ average time between electron detections, commensurate with typical photoion TOFs. In order to accommodate such ionization rates, Bodi et al. introduced a multiple-start/multiple-stop

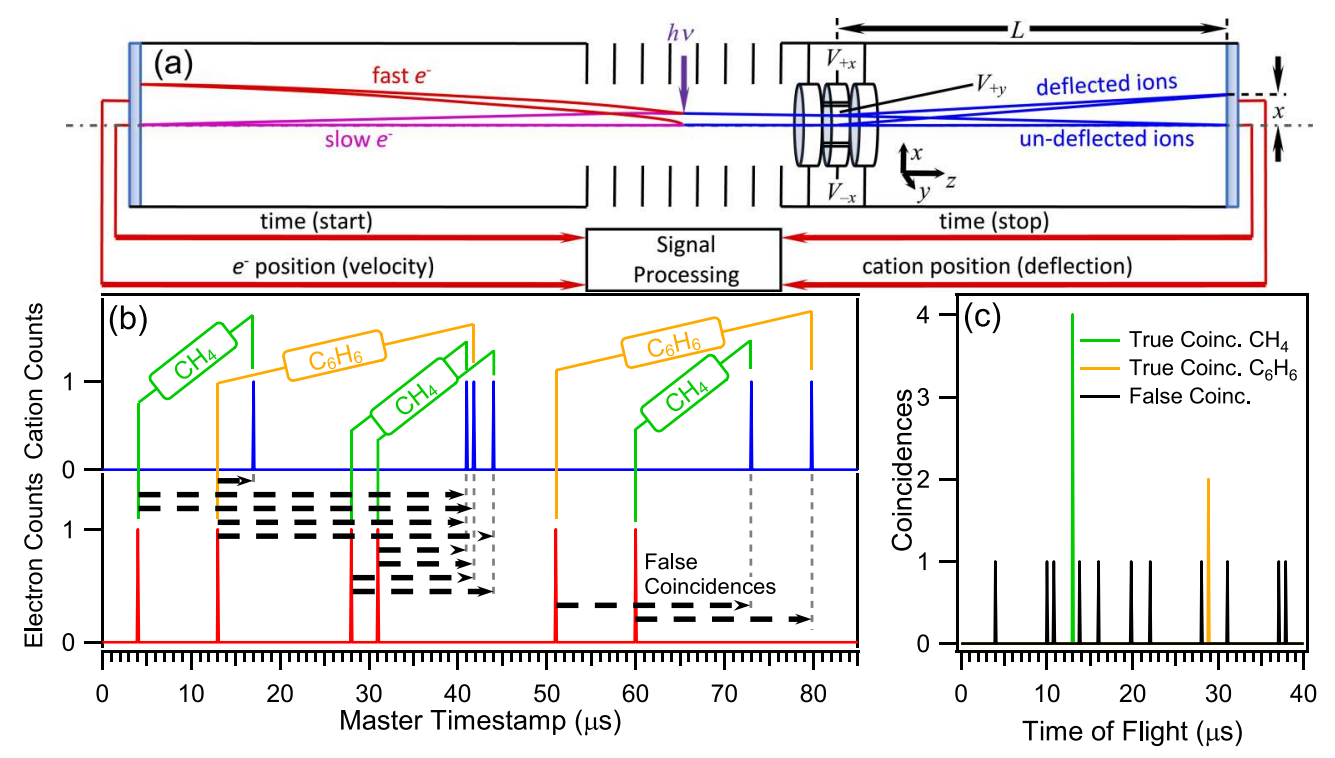

FIG. 1. (a) Schematic of the PEPICO experiment. The molecular beam (MB) propagates along the $y$ axis, crossing the photon beam (purple) at the center of the chamber. The three-element ion deflector is at the beginning of the cation flight tube on the right side. Charged particles are detected by fast position-sensitive detectors (blue rectangles). The observables measured are shown on the red arrows, with the information they carry given in parentheses. (b) Simulated time record for a two-component sample, with electron (red) and cation (blue) detection events, assuming $100 \%$ detection efficiency. Using a $40 \mu$ s time window, the six true coincidences are labeled for methane (green) and benzene (orange). Eleven pairs that form false coincidences are connected by black dashed lines. (c) The corresponding time-of-flight spectrum of coincidence events, with true coincidences in color and false coincidences in black. 
(MS/MS) coincidence data acquisition scheme, in which all electron and ion events are timestamped by a master clock. As discussed below, each photoelectron and photoion pair with detection times separated by less than an appropriately chosen time difference is tagged as a coincidence event (either a true or a false coincidence). ${ }^{21}$

Figure 1(b) illustrates the timing information in the data stream at $C=10^{5} \mathrm{~s}^{-1}$ total ionization rate and $100 \%$ detection efficiency of electrons and photoions. In this case, the rate of true coincidences is equal to the ionization rate $C$. (In reality, the true coincidence rate is linearly proportional to both the electron and the ion collection efficiencies.) Note that in Fig. 1(b), the abscissa is not the TOF, but the absolute detection time of electrons and photoions from the master clock. For each electron-ion coincidence event, the photoion's time of flight is simply calculated as $\mathrm{TOF}=($ ion timestamp - electron timestamp). Because there is no a priori information on which electron belongs to which ion, a coincidence event is tabulated for each electron/ion pair separated by less than a judiciously chosen timeof-flight difference $(\Delta t<40 \mu \mathrm{s}$ in Fig. 1). This window length $\Delta t$ is chosen to be slightly longer than the TOF of the heaviest expected cation. The simulation in Fig. 1(b) depicts four ionization events of $\mathrm{CH}_{4}(\mathrm{TOF}=13.0 \mu \mathrm{s})$ and two ionization events of $\mathrm{C}_{6} \mathrm{H}_{6}(\mathrm{TOF}=28.8 \mu \mathrm{s})$. The histogram in Fig. 1(c) displays the TOF spectrum of the 17 coincidence events (6 true and 11 false) from this data stream. At this count rate, the false coincidences outnumber true coincidences by a factor of two, but only the latter carry useful information.

At low count rates, specifically $C<<(\Delta t)^{-1}$, the time difference between photoion arrivals is sufficiently long so that only the electron/ion pair born from a single neutral is likely to be detected within the TOF window $\Delta t$, and false coincidences are negligible. At high count rates, $C>(\Delta t)^{-1}$, false coincidences become increasingly likely because several photoions may be detected within time $\Delta t$ following each photoelectron, only one of which (at most) can be the true coincident photoion. In Fig. 1(b), the 11 false coincidences are elucidated by black dashed lines connecting unrelated electron-ion pairs that satisfy TOF $<\Delta t$. Because the false coincidence rate is proportional to both the electron and ion count rates, it scales with the square of the ionization rate, $C^{2}$, and forms a flat background of equal intensity at all $\mathrm{m} / \mathrm{z}$ when using the MS/MS data acquisition technique. At sufficiently high count rates, the majority of all counts in the TOF spectrum are false coincidences, as demonstrated in Fig. 1(c). In contrast, true coincidences create narrow peaks (at integer $\mathrm{m} / \mathrm{z}$ ), whose height above the false coincidence background is proportional to $C$. $^{21}$

Although one can subtract the average false coincidence background from the TOF spectrum, the Poisson noise on this background remains and is proportional to $\sqrt{C^{2}}$ $=C$. Consequently, both the noise in the false-coincidence background and the true coincidence peak height increase linearly with total count rate. This predicament is a classic Catch-22 situation: ${ }^{22}$ it is possible to reduce false coincidences noise by decreasing the ionization rate, but this change decreases the true coincidence level by exactly the same amount, yielding no improvement in signal-to-noise. Because the Poisson noise on the false coincidence background increases with the square root of the integration time, whereas the true coincidence signal is a linear function thereof, the signal-to-noise ratio can be doubled by measuring four times longer. This approach offers nugatory benefits and is hardly ever a feasible option, because the available experimental time, the stability of the precursor compounds, or even the lifespan of the scientist do not allow one to increase the integration time by a factor 10000 in order to improve the dynamic range by two orders of magnitude. This phenomenon constitutes the false coincidence barrier, which inherently limits the dynamic range, and thus the analytical utility of PEPICO.

A high dynamic range of $10^{5}$ or greater is often required for the observation of reactive intermediates in chemically reacting mixtures, where it is normal for one molecule, most commonly an excess reactant, to be present in vastly higher concentration than other, usually more interesting species. In this case, almost all false coincidences arise from photoions of the dominant species. Therefore, the solution must lie in identifying false coincidences and removing them from the dataset. Both the TOF spectrum and the photoelectron spectrum correlated with each minor $m / z$ peak will then have improved signal-to-noise.

In this paper, we report a novel method for removing false coincidences by introducing an additional spatial dimension of detection for each cation. By deflecting cations with a known, time-dependent electric field, the arrival position of an ion can be predicted based on its absolute deflection time, which is calculated from its detection timestamp and its measured TOF, with all timing events referenced to a single master clock. A false coincidence will have an ion position inconsistent with its apparent TOF, allowing us to eliminate it from the dataset. To further increase the selectivity of false-coincidence tagging, ions can be deflected in both dimensions of the detector plane, with the additional advantage of evenly spreading the ion load on the microchannel plates, increasing detector lifetime, while avoiding localized gain reductions across the detection area.

We note that PEPICO spectroscopy has also been implemented with pulsed laser photoionization sources. ${ }^{23-26}$ However, we restrict the discussion to continuous or quasicontinuous sources of VUV radiation, such as discharge lamps or synchrotron radiation, because they are better suited to quantitative analysis.

\section{METHODS}

To demonstrate this approach, we built a prototype photoelectron photoion coincidence spectrometer that leverages existing infrastructure at the VUV beamline of the Swiss Light Source (SLS). ${ }^{19,27}$ Here, we only describe in detail the experimental aspects germane to false coincidence background rejection.

Pure argon gas was expanded into the source chamber along the $y$-axis (Fig. 1(a)) via a $50 \mu \mathrm{m}$ orifice from a molecular beam source with a stagnation pressure of 0.8 bar. The pressure in the source chamber was kept below 
$2 \times 10^{-4}$ mbar. The beam passed through a $2 \mathrm{~mm}$ skimmer before entering the ionization chamber, where the pressure was maintained at less than $6 \times 10^{-6}$ mbar. Single photon ionization is achieved using a monochromatic vacuum UV synchrotron beam with less than $2 \times 2 \mathrm{~mm}$ cross section. Electrons and ions are extracted and accelerated in opposite directions using a $250 \mathrm{~V} \mathrm{~cm}^{-1}$ constant electric field at the ionization spot. Both electrons and ions are velocity map imaged in this double-imaging spectrometer onto separate DLD40 Roentdek position-sensitive delay-line detectors. For threshold photoelectron spectroscopy, the photoelectron detector has better than $1 \mathrm{meV}$ resolution at near zero kinetic energy. Photoions pass through a two-stage grid-less Wiley-McLaren TOF mass spectrometer with a $25 \mathrm{~mm}$ long first and an $82 \mathrm{~mm}$ long second acceleration region, and an $890 \mathrm{~mm}$ long drift region, satisfying both space-focusing and velocity map imaging conditions. Argon ions formed in the molecular beam are imaged onto a spot with FWHM between 0.8 and $0.9 \mathrm{~mm}$.

As shown in Fig. 1(a), $66 \mathrm{~mm}$ after the start of the ion drift region, photoions enter an einzel-lens-style deflector assembly. The first and third elements are cylindrical $60 \mathrm{~mm}$ long, $60 \mathrm{~mm}$ inner diameter electrodes at flight tube potential. The central element is identical except that it is $24 \mathrm{~mm}$ long and is segmented into four $90^{\circ}$, individually controlled electrodes $\left(V_{+x}, V_{-x}, V_{+y}\right.$, and $\left.V_{-y}\right)$ to deflect the ions along the $x$ and $y$ axes. The $x$ and $y$ pairs of electrodes are each biased antisymmetrically, with their average potential equal to the flight tube voltage. To calibrate an ion's detected position vs. deflection voltage, ions can be deflected statically by DC voltages applied to the four deflector plates (see Fig. S1(c) of the supplementary material).

In the false coincidence suppression scheme, ions are deflected dynamically using triangle waveforms produced by a Keysight 33512B two-channel arbitrary waveform generator. These waveforms are amplified by Thorlabs HVA200 high voltage amplifiers. The output $V_{+x}, V_{-x}$, and $V_{+y}, V_{-y}$ voltage pairs are then AC-coupled to the flight tube high voltage. The full $\pm 200 \mathrm{~V}$ swing available with the HV amplifiers corresponds to a deflection of about $\pm 20 \mathrm{~mm}$ on the Roentdek DLD40 detector, ideal for its size.

The triangle waveforms, with frequencies of $18-100 \mathrm{kHz}$, have an intentional $1 \%-20 \%$ frequency detuning between the $x$ and $y$ waveforms. A deflection cycle consists of 20-100 periods of the higher-frequency triangle wave. The four triangle waves are synchronized to $0 \mathrm{~V}$ at the beginning of a deflection cycle $\left(t_{\text {cyc }}=0\right)$, which is marked by a synchronization pulse from the arbitrary waveform generator and is digitized by the PEPICO data acquisition computer. Figure 2 shows a resulting ion image of a tightly focused ion spot deflected to form periodic checkered patterns. The frequency detuning between the $x$ and $y$ deflection waveforms determines the periodicity of the checkered pattern on the ion image. We used the $100 / 90 \mathrm{kHz}$ triangle wave frequencies for most of the false-coincidence suppression in this work. An even smaller detuning enables a quasi-uniform illumination of the photoion MCP detector.

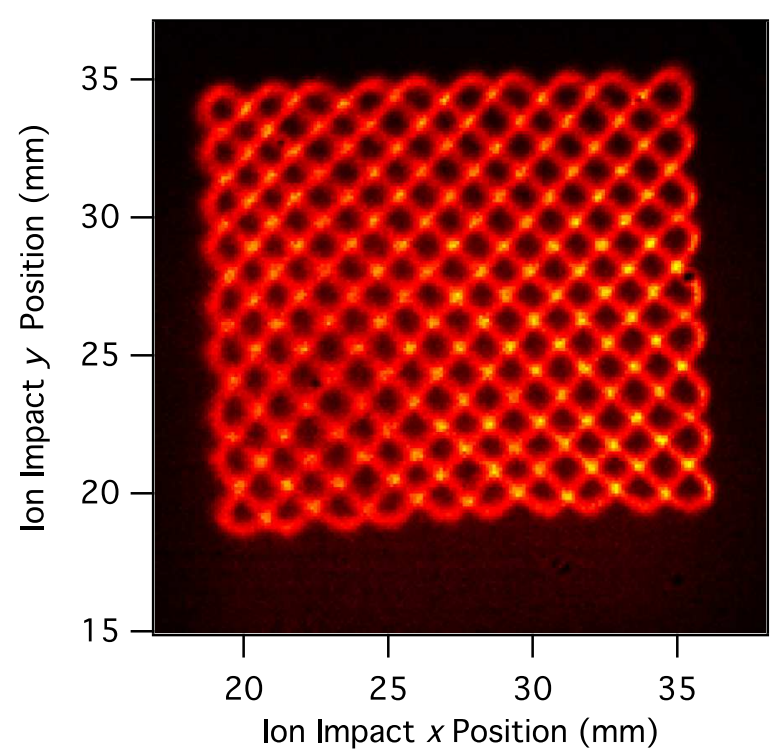

FIG. 2. PEPICO Ar${ }^{+}$image with deflection frequencies of $100 \mathrm{kHz}$ ( $x$ axis) and $90 \mathrm{kHz}$ ( $y$ axis).

In the prototype double-imaging PEPICO experiment, both the start (photoelectron) and stop (photoion) events have spatial coordinates $(x, y)$ in addition to timestamps. Two types of photoelectron spectra can be obtained in imaging PEPICO, enabled by the $(x, y)$ electron coordinates. Threshold photoelectron spectra can be collected by scanning the photon energy, retaining only coincidence events corresponding to zero kinetic energy photoelectrons, which appear in the VMI image center spot, with appropriate subtraction of energetic electrons. ${ }^{15,28}$ Alternatively, the electron image at any photon energy can be reconstructed to obtain the total photoelectron spectrum or the photoion mass-selected photoelectron spectrum and anisotropy parameters. ${ }^{29-31}$ However, as the effect of the initial electron kinetic energy on its time of flight is negligible, it does not affect the false coincidence suppression technique presented herein, and we only discuss the ion spatial coordinates hereafter.

\section{RESULTS}

The supersonic expansion yields translationally cold argon atoms and clusters with a common high velocity and narrow velocity spread. For ionization events that do not involve fragmentation of the ion, the VMI ion image is therefore a small spot with FWHM $=0.8-0.9 \mathrm{~mm}$ (Figs. S1(a) and S1(b) of the supplementary material), resulting from momentum imaging the small velocity spread of the neutrals. However, multiplexed photoionization mass spectrometry (MPIMS) experiments probing chemical reactions often involve sampling from a low-pressure reactor, and the resulting subsonic expansions are warmer along the beam propagation direction ( $y$ axis), leading to a slightly elongated ion spot. Depending on the expansion parameters, this difference may lead to a lower dynamic range gain than in the supersonic expansion data presented here. In the worst case, with no expansion-driven cooling, we can realistically 
assume one order of magnitude less gain in the dynamic range.

We now present the procedure, followed by a detailed explanation, for identifying false coincidences, applied to all electron/ion pairs in the raw data stream for which TOF $<\Delta t$.

(1) Calculate TOF $=\left(t_{\text {ion }}-t_{\text {elec }}\right)$, where $t_{\text {ion }}$ and $t_{\text {elec }}$ are the timestamps of ion and electron detection.

(2) Based on the TOF and the experimental geometry (parameter $\eta$, vide infra), calculate the time at which the ion was deflected $\left(t_{\text {deff }}\right)$.

(3) Retrieve the $V_{ \pm x}$ and $V_{ \pm y}$ voltages at $t_{\text {defl }}$ and convert these voltages to the predicted ion impact coordinates $\left(x_{\text {calc }}\right.$, $y_{\text {calc }}$.

(4) Compare the measured ( $\left.x_{\text {expt }}, y_{\text {expt }}\right)$ ion impact coordinates with the calculated ones and compute the distance between these two points, $\Delta r$.

(5) If the deviation $\Delta r$ is greater than a predefined tolerance, assign the event as a false coincidence and remove it from the dataset. Otherwise, retain the event as a true coincidence and add one event to the corresponding bin in the TOF spectrum.

The $(x, y)$ arrival position of a deflected ion at the imaging detector is determined by the voltage on the deflection plates at the time $\left(t_{\text {deff }}\right)$ that the ion passed through the deflector assembly. SIMION simulations of ion trajectories with modulated deflection fields confirm that $t_{\text {defl }}$ is uniquely determined by

$$
t_{\text {defl }}=t_{\text {elec }}+\mathrm{TOF} \times \eta,
$$

where $\eta$ is the fraction of TOF needed for any ion to travel from its birth position to the effective center of the central deflector element. The effective center is the point in the deflector that acts as if the ions were instantaneously given a perpendicular kick proportional to the deflection voltage. Under our conditions, the experimentally determined value for $\eta$ is 0.303 . For convenience, this equation can be recast in terms of the photoion timestamp,

$$
t_{\text {defl }}=t_{\text {ion }}-(1-\eta) \times \mathrm{TOF},
$$

where the value $(1-\eta)=0.697$ is the fraction of the TOF needed for any ion to travel the distance $L$ (Fig. 1(a)) from the point of deflection to the detection plane. A critical fact is that $\eta$ is independent of photoion mass. As an example, the first true coincidence shown in the simulation of Fig. 1(b) is from a $\mathrm{CH}_{4}$ molecule $(m / z=16)$. It is characterized by $t_{\text {elec }}=4.000 \mu \mathrm{s}$, $\mathrm{TOF}=(17.000-4.000)=13.000 \mu \mathrm{s}$, and hence it would be deflected at a master timestamp of $t_{\text {defl }}=7.939 \mu \mathrm{s}$. Using the recorded timestamp $t_{\mathrm{cyc}}$ of the most recent sync pulse from the arbitrary waveform generator, we can retrieve the magnitudes of the four voltages at the time of deflection: $V_{ \pm x}\left(t_{\mathrm{defl}}-t_{\mathrm{cyc}}\right)$ and $V_{ \pm y}\left(t_{\text {defl }}-t_{\text {cyc }}\right)$. As mentioned earlier, SIMION simulations show that the $(x, y)$ coordinates of the ion impact position are uniquely determined by the voltages on the deflector plates at the time of deflection. The relationship between the ion impact coordinates and deflection voltages is initially obtained from static deflection of ions. The $\underline{V} \rightarrow \underline{r}_{\text {calc }}$ transformation parameters are then optimized on the dynamic-deflection experimental data itself. In the prototype experiment, where the ion image center does not coincide with the detector center and the deflection axes are not exactly parallel with the image axes, the following linear transformation converts the deflection voltage vector $\underline{V}$ to the ion impact position vector:

$$
\underline{r_{\text {calc }}}=\underline{r_{0}}+\underline{V} \cdot\left(\begin{array}{cc}
P_{1} & P_{2} \\
P_{3} & P_{4}
\end{array}\right),
$$

where $r_{0}$ is the offset of the image center, and $P_{1-4}$ are the matrix elements of the linear transformation. (One way to think about these four parameters is that two describe multiplicative scaling in two dimensions, one corrects the image rotation and the last parameter corrects for skew if the angle between the $x$ and $y$ deflection axes is not a right angle.) The distance $\Delta r$ between the measured coordinates of ion impact, $\left(x_{\text {expt }}, y_{\text {expt }}\right)$, and the calculated position ( $\left.x_{\text {calc }}, y_{\text {calc }}\right)$ is simply

$$
\Delta r=\sqrt{\left(x_{\text {expt }}-x_{\text {calc }}\right)^{2}+\left(y_{\text {expt }}-y_{\text {calc }}\right)^{2}} .
$$

In the case of a true coincidence, $\Delta r$ will be less than a chosen tolerance $\varepsilon$, which is roughly the radius of the photoion spot at the detector. A false coincidence, arising from an unrelated electron/ion pair, will almost always be characterized by $\Delta r>\varepsilon$.

To understand why this is so, recall that $\eta$ is independent of mass. The time needed for any ion to travel from the deflector to the detector is always a fixed fraction of its total TOF (69.7\% in our apparatus). For a false coincidence, the calculated TOF value is unrelated to the actual ion mass and, consequently, the assumption that the ion covered the distance $L$ in $69.7 \%$ of this TOF should not hold. Therefore, the calculated value of $t_{\text {defl }}$ is, in fact, not the true time this ion passed through the central deflector. Hence the retrieved deflector voltages are not the actual voltages that deflected this ion, and therefore the calculated position of impact $\left(x_{\text {calc }}, y_{\text {calc }}\right)$ does not agree with the measured impact position $\left(x_{\text {expt }}, y_{\text {expt }}\right)$, resulting in $\Delta r>\varepsilon$. By varying $\varepsilon$, one controls the degree of false coincidence rejection.

Computer simulations of false coincidence suppression, assuming ideal ion spot sizes and realistic time-of-flight peak widths, predict a dynamic range of better than $10^{5}$. These simulations, presented as supplementary material, clearly show that simple background subtraction is inadequate to increase dynamic range. Guided by these results, we designed and tested the prototype PEPICO apparatus, in which a supersonic molecular beam sample of pure argon gas was ionized. $\mathrm{Ar}^{+}$provided the dominant TOF peak, whereas the neutral dimer, formed in the cold expansion, yielded $\mathrm{Ar}_{2}{ }^{+}$ upon ionization, playing the role of the minor coincidence signal on which the improved dynamic range can be judged.

The data analysis consists of two steps: parameter optimization and false-coincidence suppression. The optimization (1) establishes the transformation between deflector voltages and image coordinates, (2) fits the experimental value for $\eta$, and (3) corrects for any small variation in the experimental sweep frequency, which arises because the waveform generator and the electron/ion data collection card have independent timebases. Because the $\mathrm{Ar}^{+}$peak is overwhelmingly composed of true coincidences, we can focus on its narrow TOF 
range to optimize the transformation parameters for minimum average deviation between the experimental and calculated ion positions. The derived parameters are used in the falsecoincidence suppression to reject coincidences where the theoretical and actual positions do not agree to within $\Delta r<\varepsilon$.

We now discuss some effects on our analysis caused by the molecular beam (MB) source of argon. First, in these measurements, both true and false coincidences are dominated by $\mathrm{Ar}^{+}$ions from the molecular beam, whereas other ions are lower in abundance by several orders of magnitude. This means that coincidence events at times-of-flight different from that of $\mathrm{Ar}^{+}$will most likely be false coincidences. Second, all species in the supersonic beam move with the average velocity of the carrier gas. Hence, the more atoms in an argon cluster, the greater its momentum, resulting in each cluster size being imaged to a slightly different spot on the detector, which was accounted for in the data analysis. Third, there is a thermal background of mostly Ar atoms, which have greater velocity spread but much lower velocity along the MB axis and, therefore, appear offset from $\mathrm{Ar}^{+}$ionized from the cold molecular beam. Bearing these points in mind, Fig. 3 shows the experimental spatial positions of the ions in the $x$ (red) and $y$ (blue) directions as a function of time since the last deflection sync pulse. Coincidence events with a TOF corresponding to $\mathrm{Ar}^{+}$are plotted with open circles, whereas events characterized by other TOF values are shown with a dot. For comparison, the calculated ion positions for true coincidence events are shown as solid lines, with $x_{\text {calc }}=y_{\text {calc }}=0$ at $t=0$ and $50 \mu \mathrm{s}$ (corresponding to 5 and 4.5 periods, respectively). Note that if there were no false coincidences nor thermal background, all data in Fig. 3 would lie on the black and blue triangle waves. In the $x$ deflection direction (red circles), perpendicular to the $\mathrm{MB}$ axis, most of the $\mathrm{Ar}^{+}$ions are either at their expected positions or are scattered closely above and below the true coincidence line. Whereas, along the $y$ deflection direction (blue circles, deflection parallel to the MB axis), most of

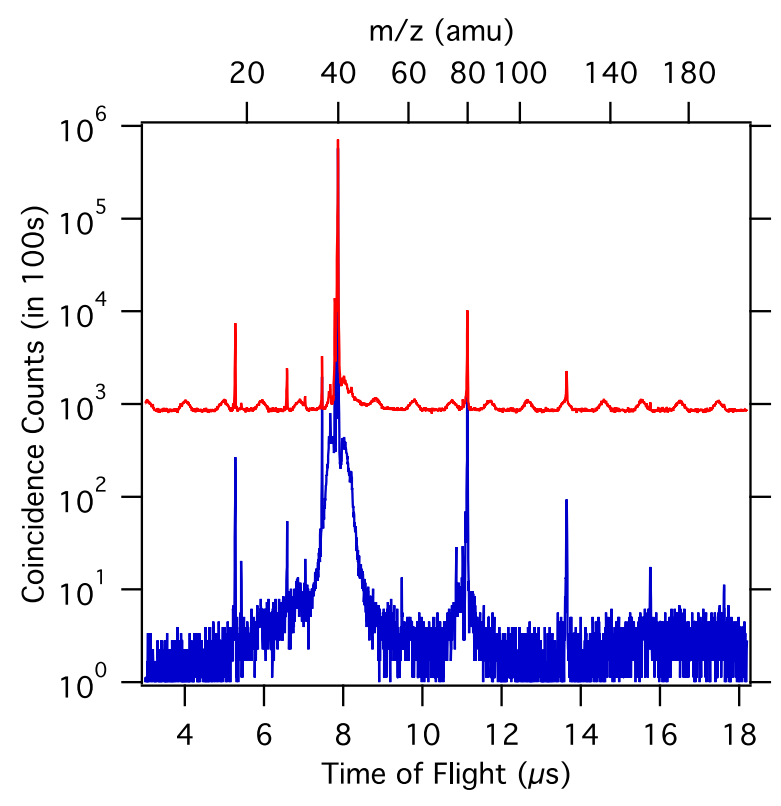

FIG. 4. Time-of-flight coincidence spectra from the Ar molecular beam experiments. The top curve (red) is the original coincidence spectrum, whereas the lower curve (blue) shows the result of false-coincidence suppression. Note the logarithmic scale.

the $\mathrm{Ar}^{+}$ions not on the line are systematically at lower positions: these are the thermal, slower argon ions, vide supra. However, most coincidences that are off the reference lines have a TOF other than that of $\mathrm{Ar}^{+}$(dots instead of circles) and these are overwhelmingly false coincidences. In the end, ions with positions more than $\pm \varepsilon$ away from these triangle waves have a position that disagrees with their time of flight and are therefore tagged as false coincidences and discarded.

Raw and false-coincidence suppressed time-of-flight spectra at a $5.5 \times 10^{4} \mathrm{~s}^{-1}$ total coincidence count rate are shown in Figure 4. The false coincidence level was roughly 850 counts in each $5 \mathrm{~ns}$ time bin after $100 \mathrm{~s}$ of total measurement

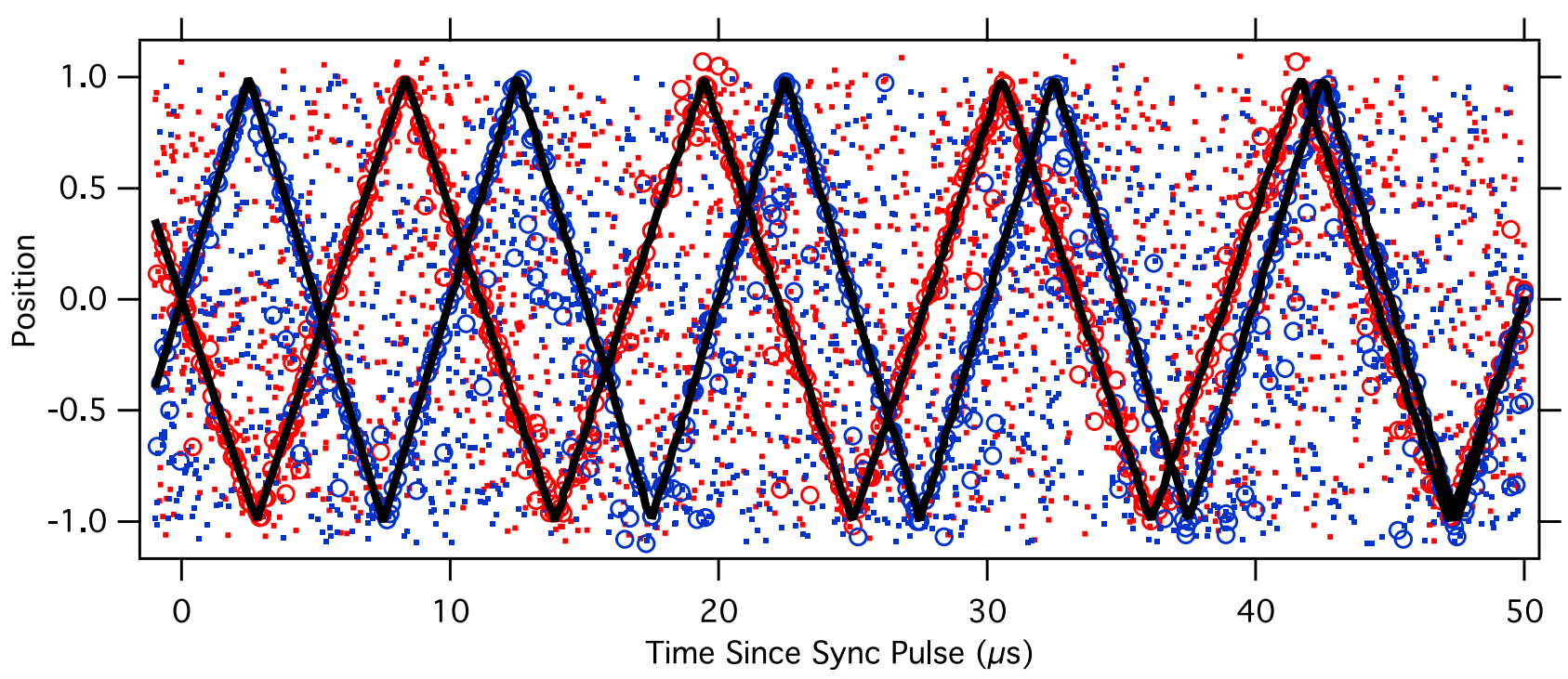

FIG. 3. Normalized ion positions parallel (blue) and perpendicular (red) to the molecular beam. Open circles correspond to coincidences with Ar ${ }^{+}$time of flight, while solid dots indicate coincidences with time of flight other than that of $\mathrm{Ar}^{+}$. The calculated nominal positions based on time since the sync pulses are shown with solid lines. 


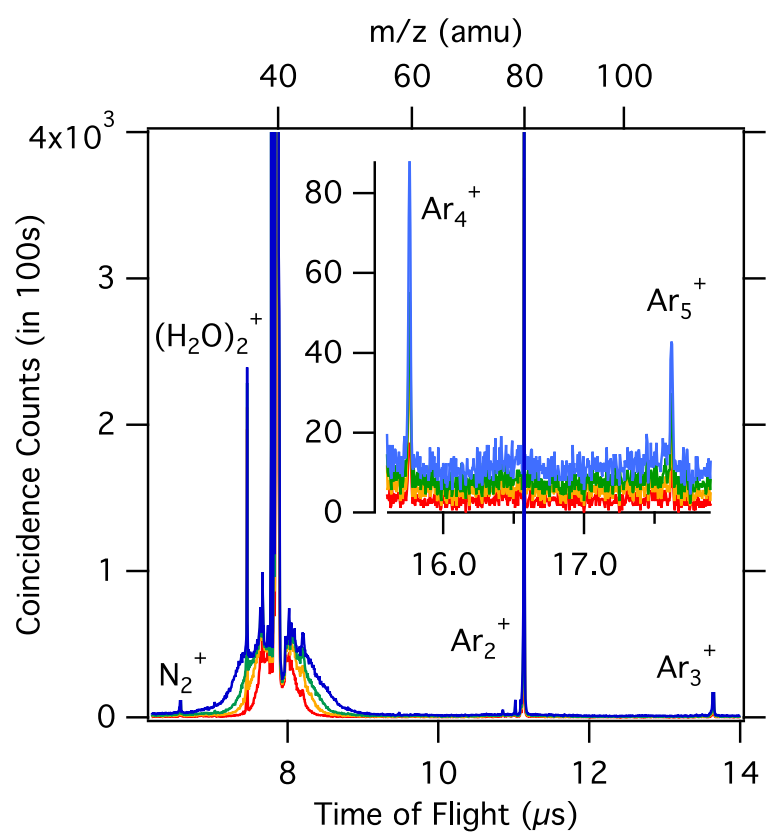

FIG. 5. False-coincidence suppressed time-of-flight coincidence spectra with four different true-coincidence acceptance radii. The highest intensity trace (purple) corresponds to $\varepsilon=4 \mathrm{~mm}$, whereas the lowest intensity trace (red) corresponds to $\varepsilon=0.8 \mathrm{~mm}$. Note that the pedestal surrounding $7.9 \mu \mathrm{s}$ is hardly visible when the ordinate is scaled to show the full peak height of $\mathrm{Ar}^{+}$.

time. The false coincidence signal is not completely flat: it exhibits periodic peaks of $30 \%$ in amplitude at a $0.96 \mu \mathrm{s}$ period, corresponding to the time structure of the synchrotron radiation. (The filling pattern of the synchrotron is such that a ca. $560 \mathrm{~ns}$ bright period is followed by a $400 \mathrm{~ns}$ dark gap. This structure modulates the uncorrelated electron and ion flux, resulting in a false coincidence background that oscillates slightly with TOF.) The false coincidence suppressed TOF spectrum shows an almost $10^{3}$ decrease in the background and complete suppression of the periodic false-coincidence peaks resulting from the synchrotron time structure.

We have explored the effect of varying the radius of acceptance, $\varepsilon$, in false coincidence suppression. Results with $\varepsilon=0.8,1.6,2.4$, and $4 \mathrm{~mm}$ are plotted in Figure 5. To interpret this effect, consider the following three aspects. (1) As shown in the inset of Figure 5, at small $\varepsilon$ values, true coincidences are also rejected and the heights of the peaks in the spectrum are diminished as useful information is discarded. (2) Also from the inset, larger $\varepsilon$ increases the flat false coincidence background at TOF values far from the dominant peaks, because some of the false coincidences happen to fall within the radius of acceptance and are not rejected. These two factors constrain the ideal acceptance radius to maximize the dynamic range when the low-intensity peaks are far from the dominant one(s) in the mass spectrum. (3) Around the dominant $\mathrm{Ar}^{+}$ peak, there is a pedestal of false coincidences, the width of which increases with increasing $\varepsilon$, as shown in Figure 5. This pedestal is due to false coincidences with an apparent time of flight that is very close to the dominant $\mathrm{Ar}^{+}$peak.

To understand why these false coincidences in the pedestal are not removed, let us first discuss the set of false coincidences with apparent time-of-flight values far from that of the dominant peak. Because the stop signals in this set of false coincidences are overwhelmingly created by the dominant ion $\left(\mathrm{Ar}^{+}\right.$in this case), their apparent time of flight values are very different from those of the true $\mathrm{Ar}^{+} \mathrm{TOF}$. Therefore the calculated deflection time (and voltages) will be substantially different than the case of a true $\mathrm{Ar}^{+} / \mathrm{e}^{-}$coincidence event, and the calculated and measured ion positions clearly disagree. By contrast, a false coincidence with a "false" time of flight very close to the true time of flight of the ion involved will result in a calculated deflection position that is close to the experimental value, and this false coincidence may not be rejected. For example, at $100 \%$ collection efficiencies, consider two $\mathrm{Ar}^{+}$ions whose birth times differ by only $10 \mathrm{~ns}$. These events create two false and two true coincidences, but the deflection voltages have barely changed over $10 \mathrm{~ns}$. At realistic (i.e., $<100 \%$ ) collection efficiencies, it is possible that only the false (or only the true) coincidences are detected. The smaller the $\varepsilon$, the closer to the dominant peak we can still distinguish false coincidences from the true ones. In practical terms, this means that false coincidences beneath the dominant peak(s) are poorly suppressed, and $\varepsilon$ determines the width of the false-coincidence pedestal around such very large peaks. This trade-off plays an important role and determines the optimal $\varepsilon$ when the minor peak of interest is near a dominant peak.

Generally speaking, the dynamic range gain depends on the parent ion spot size divided by the active area of the detector. Decreasing the photoion spot size at the detector and increasing the area over which ions impact the detector make it less likely that the theoretical and measured ion positions accidentally agree for a false coincidence. The conditions for false coincidence suppression are least favorable when the sample temperature is high, but we expect that a dynamic range increase of $10^{2}$ will always be within reach, particularly by raising the extraction field to improve ion focusing and certainly by using a larger imaging detector. As for the suppression of false coincidences near the dominant peak in the spectrum, the relationship between the distance in spatial position on the detector and the difference in deflection time is established by the sweep rate of the deflection voltages. Faster sweep rate (that is, the product of the sweep frequency and the image size) translates to better spatial separation on

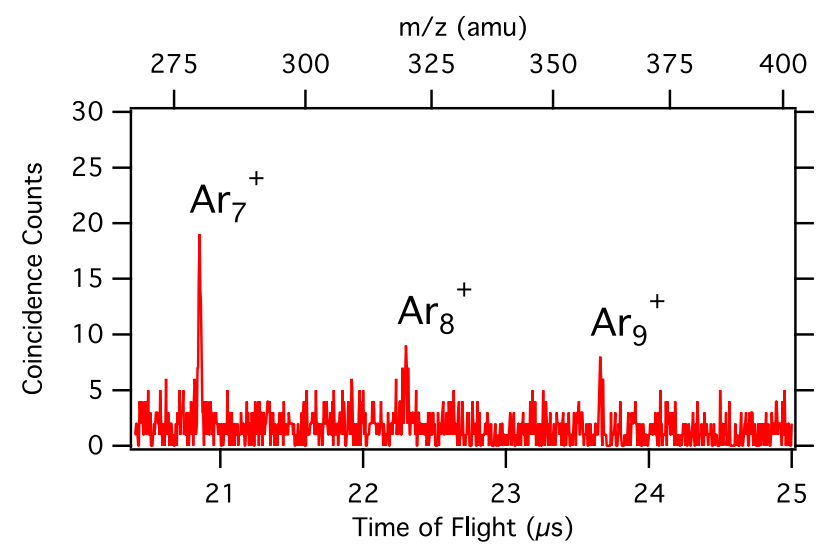

FIG. 6. False-coincidence suppressed time-of-flight coincidence spectrum of the larger argon oligomer ions. 
the ion image, making the false-coincidence suppression more effective, especially close to the dominant time-of-flight peak.

Finally, we would like to illustrate the power of this novel false coincidence suppression method on argon oligomer ions not previously detected in coincidence experiments. Depending on the backing pressure and the resulting supersonic cooling, the SLS iPEPICO apparatus is able to detect $\mathrm{Ar}_{3}{ }^{+}$or, under ideal conditions, even $\mathrm{Ar}_{4}{ }^{+}$. Larger oligomers, however, have signals so small that they are usually obscured by the false coincidence background. However, Fig. 6 shows that the prototype apparatus, together with false coincidence removal, allows detection of much larger neutral argon oligomers with correspondingly lower populations, including the argon nonamer ion, $\mathrm{Ar}_{9}{ }^{+}$.

\section{CONCLUSIONS}

We have shown that the false coincidence barrier, which limits the dynamic range of conventional PEPICO experiments, can be overcome with ion imaging used with a swiftly modulated electric deflection field acting on the ion trajectories at a fixed fraction of their time of flight. Most false coincidences obscuring minor peaks in the mass spectrum arise from the most dominant ions. Because the calculated TOF for a false coincidence is erroneous, the calculated time of ion deflection is also incorrect, leading to a predicted impact position that does not agree with the observed impact position. As a result, only true coincidences will be detected at the predicted impact position, allowing the false coincidence background to be removed, thereby increasing the dynamic range of the experiment by at least two orders of magnitude. Proof-of-principle supersonic molecular beam spectra of argon illustrate how parent cations from $\mathrm{Ar}_{9}$ oligomers can be easily detected using this false coincidence suppression scheme, even when $\mathrm{Ar}_{4}$ species are hardly detectable without suppression. Eliminating false coincidences breaks down the major barrier to utilizing PEPICO as a sensitive and versatile analytical technique, offering photoelectron spectral fingerprints of even very minor and transient components of a time-evolving, reactive mixture.

\section{SUPPLEMENTARY MATERIAL}

The supplementary material includes a brief description of the calibration of the ion impact position using static deflections, including an image of the undeflected ion spot and both $x$ and $y$ cross sections thereof. An ion image using appropriately chosen ion spot locations and pixel dwell times is also given. The dynamic deflection of ions is further illustrated with two more ion images using different $x / y$ waveform frequency ratios. Finally, a section on the in silico simulation of the false coincidence suppression method shows time-offlight PEPICO spectra of two peaks with a $10^{5}$ intensity ratio, both with or without false coincidence suppression.

\section{ACKNOWLEDGMENTS}

This work has been funded by the National Science Foundation (Grant No. CHE-1266407) and by the U.S.
Department of Energy under the Visiting Faculty Program. The experimental work was carried out at the VUV beamline of the Swiss Light Source of the Paul Scherrer Institute. The financial support of the Swiss Federal Office for Energy (BFE Contract Nos. 101969/152433 and SI/501269-01) is gratefully acknowledged. This material is based upon work supported by the U.S. Department of Energy, Office of Science, Office of Basic Energy Sciences.

${ }^{1}$ D. L. Osborn, P. Zou, H. Johnsen, C. C. Hayden, C. A. Taatjes, V. D. Knyazev, S. W. North, D. S. Peterka, M. Ahmed, and S. R. Leone, Rev. Sci. Instrum. 79, 104103 (2008).

${ }^{2}$ O. Welz, J. D. Savee, D. L. Osborn, S. S. Vasu, C. J. Percival, D. E. Shallcross, and C. A. Taatjes, Science 335, 204 (2012).

${ }^{3}$ J. D. Savee, E. Papajak, B. Rotavera, H. Huang, A. J. Eskola, O. Welz, L. Sheps, C. A. Taatjes, J. Zador, and D. L. Osborn, Science 347, 643 (2015).

${ }^{4}$ P. Hemberger, A. J. Trevitt, T. Gerber, E. Ross, and G. da Silva, J. Phys. Chem. A 118, 3593 (2014).

${ }^{5}$ R. Tuckett, J. Harvey, P. Hemberger, and A. Bodi, J. Mol. Spectrosc. 315, 172 (2015).

${ }^{6}$ A. Bodi, P. Hemberger, D. L. Osborn, and B. Sztáray, J. Phys. Chem. Lett. 4, 2948 (2013).

${ }^{7}$ A. Bodi, J. Csontos, M. Kállay, S. Borkar, and B. Sztáray, Chem. Sci. 5, 3057 (2014).

${ }^{8}$ P. Hemberger, A. Bodi, J. H. J. Berthel, and U. Radius, Chem. - Eur. J. 21, 1434 (2015).

${ }^{9}$ A. Bodi, T. Baer, N. K. Wells, D. Fakhoury, D. Klecyngier, and J. P. Kercher, Phys. Chem. Chem. Phys. 17, 28505 (2015)

${ }^{10}$ M. Steinbauer, P. Hemberger, I. Fischer, and A. Bodi, ChemPhysChem 12, 1795 (2011).

${ }^{11}$ P. Hemberger, M. Steinbauer, M. Schneider, I. Fischer, M. Johnson, A. Bodi, and T. Gerber, J. Phys. Chem. A 114, 4698 (2010).

${ }^{12}$ D. Felsmann, K. Moshammer, J. Krüger, A. Lackner, A. Brockhinke, T. Kasper, T. Bierkandt, E. Akyildiz, N. Hansen, A. Lucassen, P. Oßwald, M. Köhler, G. A. Garcia, L. Nahon, P. Hemberger, A. Bodi, T. Gerber, and K. Kohse-Höinghaus, Proc. Combust. Inst. 35, 779 (2015).

${ }^{13}$ D. H. Parker and A. T. J. B. Eppink, J. Chem. Phys. 107, 2357 (1997).

${ }^{14}$ T. Baer and Y. Li, Int. J. Mass Spectrom. 219, 381 (2002).

${ }^{15}$ B. Sztáray and T. Baer, Rev. Sci. Instrum. 74, 3763 (2003).

${ }^{16}$ G. A. Garcia, H. Soldi-Lose, and L. Nahon, Rev. Sci. Instrum. 80, 023102 (2009).

${ }^{17}$ G. A. Garcia, B. K. Cunha de Miranda, M. Tia, S. Daly, and L. Nahon, Rev. Sci. Instrum. 84, 053112 (2013)

${ }^{18}$ A. Bodi, M. Johnson, T. Gerber, Z. Gengeliczki, B. Sztáray, and T. Baer, Rev. Sci. Instrum. 80, 34101 (2009).

${ }^{19}$ A. Bodi, P. Hemberger, T. Gerber, and B. Sztáray, Rev. Sci. Instrum. 83, 083105 (2012).

${ }^{20}$ X. Tang, X. Zhou, M. Niu, S. Liu, J. Sun, X. Shan, F. Liu, and L. Sheng, Rev. Sci. Instrum. 80, 113101 (2009).

${ }^{21}$ A. Bodi, B. Sztáray, T. Baer, M. Johnson, and T. Gerber, Rev. Sci. Instrum. 78, 84102 (2007).

${ }^{22}$ J. Heller, Catch-22 (Simon \& Schuster, 1961).

${ }^{23}$ J. A. Davies, J. E. LeClaire, R. E. Continetti, and C. C. Hayden, J. Chem. Phys. 111, 1 (1999).

${ }^{24}$ V. Stert, W. Radloff, C. P. Schulz, and I. V. Hertel, Eur. Phys. J. D 5, 97 (1999).

${ }^{25}$ A. M. Rijs, M. H. M. Janssen, E. t. H. Chrysostom, and C. C. Hayden, Phys. Rev. Lett. 92, 123002 (2004).

${ }^{26}$ O. Gessner, A. M. D. Lee, J. P. Shaffer, H. Reisler, S. V. Levchenko, A. I. Krylov, J. G. Underwood, H. Shi, A. L. L. East, D. M. Wardlaw, E. T. H. Chrysostom, C. C. Hayden, and A. Stolow, Science 311, 219 (2006).

${ }^{27}$ M. Johnson, A. Bodi, L. Schulz, and T. Gerber, Nucl. Instrum. Methods Phys. Res., Sect. A 610, 597 (2009).

${ }^{28}$ T. Baer, B. Sztáray, J. P. Kercher, A. F. Lago, A. Bodi, C. Skull, and D. Palathinkal, Phys. Chem. Chem. Phys. 7, 1507 (2005).

${ }^{29}$ J. C. Poully, J. P. Schermann, N. Nieuwjaer, F. Lecomte, G. Grégoire, C. Desfrançois, G. A. Garcia, L. Nahon, D. Nandi, L. Poisson, and M. Hochlaf, Phys. Chem. Chem. Phys. 12, 3566 (2010).

${ }^{30}$ G. A. Garcia, L. Nahon, S. Daly, and I. Powis, Nat. Commun. 4, 2132 (2013).

${ }^{31}$ A. Bodi and P. Hemberger, Phys. Chem. Chem. Phys. 16, 505 (2014). 